\title{
1/||||||||||||||||||||||||||||||||||||||||||||||||||||||||||||||||'.
}

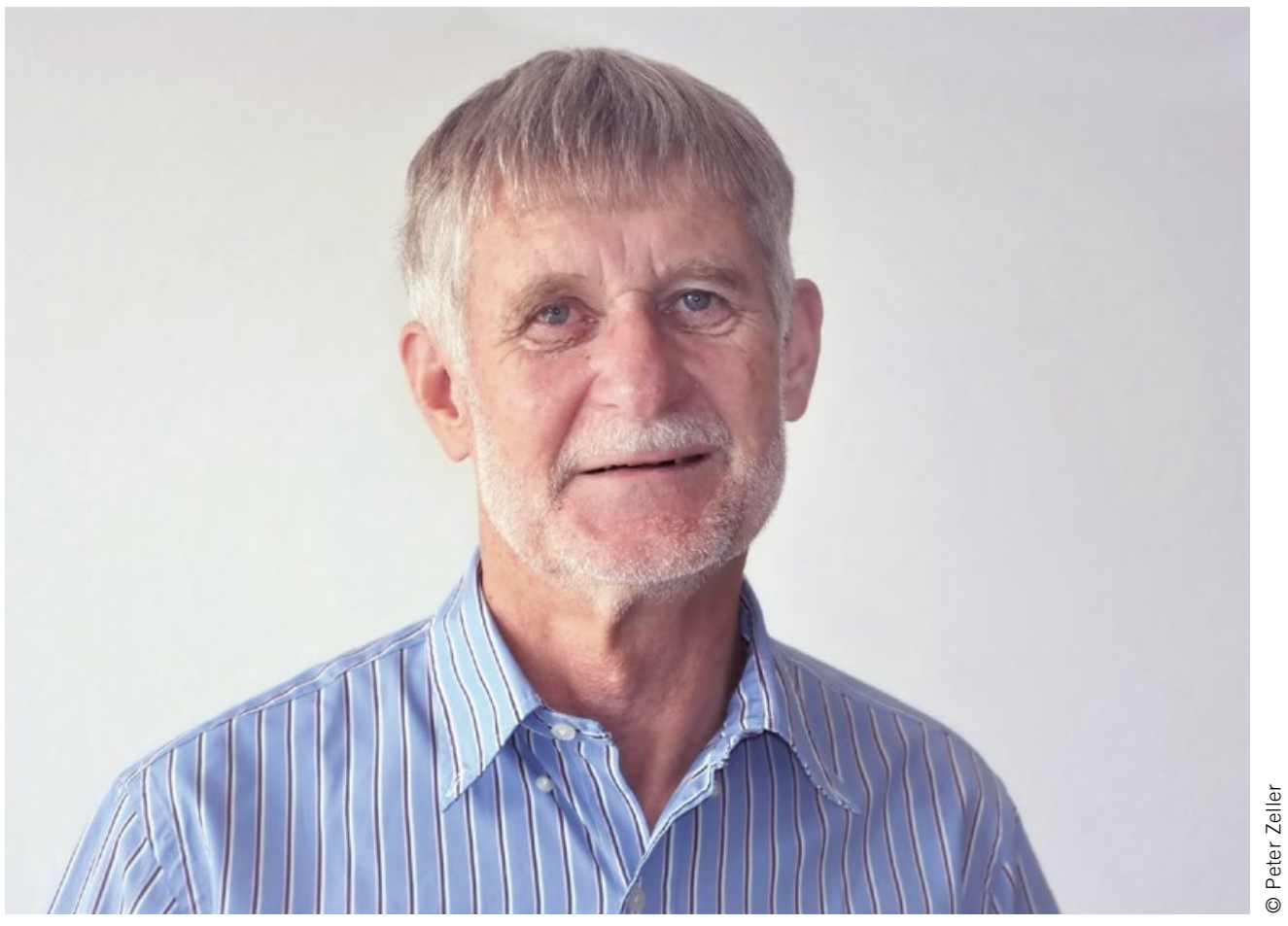

Prof. Dr.-Ing. Peter Zeller Honorary Professor at the Technical University of Munich (TUM) and Springer-Vieweg book author

\section{Maintaining Acceptance of the Car}

When we consider the ongoing public debate on the subject of the car industry, we might easily believe that we are not talking about Germany's greatest industrial success story, but about a particularly glaring case of incompetence and irresponsibility. In this hysterical discussion, we quickly lose sight of the outstanding achievements of German engineers.

At this point, I would not like to go into the question of whether and to what extent laws on vehicle exhaust emissions were broken and which institution or person made mistakes. Instead, I would like to use the example of automotive acoustics to show that, on the whole, more sensitivity is required among those responsible if our automotive success story is to continue. After all, there is hardly any other technical product that has such an impact on people's lives than the car. And that is true in both an objective and an emotional respect. For some, the car is an expression of their individual attitude towards life, whereas for others it is simply a means of transport - or even the root of all evil. It is the task of legislation to resolve this latent conflict of interests with the corresponding legal requirements. The cooperation of industry is absolutely essential in this process, as only it can introduce all of the technological and economic interdependencies. However, this also means that it has a special responsibility.

Legislation applies not only to exhaust emissions, but also to noise emissions from vehicles. In this case too, the procedure for measuring pass-by noise which was standard practice since 1982 was criticised as being barely relevant to real-world conditions, and has in the meantime been replaced by a new method that is closer to reality. EU Regulation No. 540/2014 was introduced in 2016 and imposed new vehicle noise level limits. If one brings the modified testing procedure to account, these new limits do not really impose stricter requirements regarding noise emissions. The short-term interests of the industry have prevailed over the rights of citizens to protect themselves against noise. What is also particularly problematic is the fact that the limit values have been tiered according to vehicle categories, allowing limits that are up to $4 \mathrm{~dB}$ higher for particularly powerful vehicles. In other words, the equal treatment of all cars that had applied for more than 40 years was abandoned in favour of a "noise bonus" for super sports cars. It is doubtful whether the car industry has done itself any favours in the long term with this negotiation success. The craze for synthetically programmed "misfires" to create exhaust popping, which has been around for some years now, represents another unnecessary acoustic nuisance in these vehicles. This noise is also a striking negative example of inauthentic "fake acoustics".

Only if the great accomplishments of automotive engineers are supported by a greater level of sensitivity for public welfare among those who bear responsibility will it be possible to maintain the social acceptance of the car in the years to come. 